\title{
Fracture Processes in Ductile Polymers. I. Localized Plastic Deformations of Polycarbonate and Nylon Films
}

\author{
Ikuo Narisawa, Masaru Ishikawa, and Hiroyuki Ogawa \\ Faculty of Engineering, Yamagata University, Jonan 4-3-16, \\ Yonezawa 992, Japan.
}

(Received August 1, 1975)

\begin{abstract}
The development of plastic deformation around the crack tip of known ductile polymers (polycarbonate and nylon 6) has been studied in connection with the Dugdale model of ductile yielding. It is shown that there are particular threshold stresses below which the yielding ahead of the crack tip would not be visible and that these are closely associated with the stable crack initiation. The shape of the plasticzone shows, in general, similar characteristics to those found in the Dugdale type yielding, but the size and displacement do not compare well with the Dugdale predictions. A modified Dugdale model, where the cohesive stress varies linearly with the distance from the crack tip, is then successfully applied to the analysis of the experimental data. KEY WORDS Ductile Polymer / Polycarbonate / Nylon / Yielding / Dugdale Model / Plastic-Zone / Mises Criterion / Stable Crack / Crack-Opening Displacement /
\end{abstract}

Since the pioneering work by Berry, ${ }^{1}$ who applied the Griffith theory to the fracture behavior of brittle polymers, there have been many studies to determine a fracture criterion for them. Though nearly all of these studies are essentially based on a thermodynamic approach, a somewhat different approach, called fracture mechanics, was proposed by Irwin $^{2}$ in 1957. The concept of fracture mechanics itself, although mainly developed for metals, is independent of the material to which it is applied. Recent investigations have shown, indeed, that this concept is quite successfully applicable to both the brittle crack initiation and propagation problems in different types of polymer fracture, such as static and dynamic fractures, ${ }^{3}$ fatigue, ${ }^{4}$ stress cracking, ${ }^{5}$ and crazing. ${ }^{6}$

For the linearly elastic materials, or an elastic-plastic material where the plastic deformation is small enough in comparison with the crack size, the fracture criterion obtained from fracture mechanics is the same form as that of the Griffith theory. In many practical cases, however, the majority of polymers are ductile. These polymers usually exhibit considerably greater amounts of nonlinear behavior prior to unstable fracture. Therefore, it can be of practical importance to make an examination of the applicability of linear fracture mechanics to fracture behaviors of such ductile polymers. From this point, for example, Vincent ${ }^{7}$ investigated the stable crack growth in poly(ethylene terepthalate) film to examine the limitations of linear fracture mechanics. In this study, he concluded that linear fracture mechanics could not be applied to this class of ductile polymers. Following Vincent's work, Fergunson and Williams ${ }^{8}$ have shown that Vincent's data could be correlated with a constant crack-opening displacement criterion by using the Dugdale model, ${ }^{9}$ which assumes nonlinear elasticity of the material.

The energy balance concept has been considered one of the most important methods for characterizing a fracture criterion for ductile as well as brittle materials. For a conservative system in ductile materials, one quite clearly would expect that the work put into the specimen is dissipated to create plastic deformations ahead of the crack tip. It is well known that this dissipation is frequently several orders of magnitude larger than the surface energy newly created due to a propagating crack. In order to evaluate separately the energy for plastic 
deformations at the crack tip, one should calculate the total amounts of the displacement and the stress distribution within the plastic region. Several attempts have been made to obtain these quantities experimentally in semiductile metals; however, the success is not very notable so far. If the Dugdale model can be applied to such ductile polymers, the energy balance considerations would be greatly simplified. Moreover, analytical comparisons can be made between various fracture criteria and a semi-empirical criterion, such as the crackopening displacement criterion, can gain a physical basis. Thus the Dugdale model provides the first stage of an approach to nonlinear fracture mechanics.

In these circumstances, except for the few limited attempts of Brinson $^{10}$ and Bergkvist, ${ }^{11}$ very little effort has been expended on polymers. Brinson has shown that the plastic region in polycarbonate can be identified by the photoelastic fringe shape, and he concluded that the plastic-zone size was predicted by the Dugdale model. However, it should be noted that his method cannot always be useful for determining the plastic-zone sizes of other polymers, because they are generally less sensitive to photoelasticity than is polycarbonate. Bergkvist concluded, conversely, that the plastic-zone size in poly(vinyl chloride), which was measured from displacements of an engraved grid on the specimen surface, could not compare well with the Dugdale predictions. This type of investigation has just been started so that, in this field, it is of importance to increase the experimental data at the present time, as both Brinson and Berkvist have emphasized at the end of their reports.

As the first part of the series of papers which have been undertaken to elucidate the fracture mechanism in ductile polymers, the object of this paper is to measure the plastic-zone sizes and shapes, and to compare these quantities with one of the most useful, existing nonlinear predictions.

\section{NONLINEAR FRACTURE MECHANICS (THE DUGDALE MODEL)}

Several elastic-plastic solutions with analytical and numerical methods for estimating

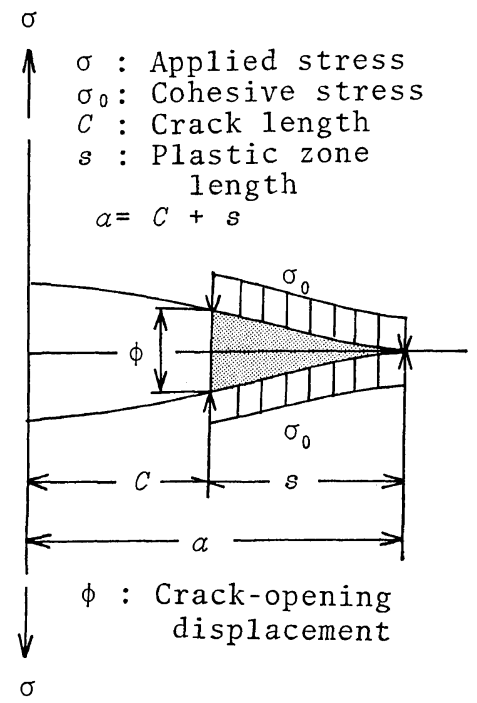

Figure 1. The Dugdale model.

the plastic-zone size have been studied. Of these, for its similicity, the Dugdale model is the most practical for a computation of the length of thin plastic-zones. As shown in Figure 1 , the assumptions of the Dugdale model for solutions are:

1. An ideal elastic-plastic material (no stress or strain hardening effects) which flows after yielding at a constant uniaxial stress $\sigma_{0}$.

2. Two separate load systems are applied on a cracked tensile sheet. One is the gross stress $\sigma$ at infinity and another is a cohesive stress $\sigma_{0}$ on each part of the crack surfaces which simulates a plastic-zone, which is a thin extension of the crack lines.

3. The plastic-zone length $s$ is such that there is no stress singularity at the crack tip.

The solution for the length of the plastic-zone which results from the above assumptions can be written.

$$
\frac{s}{a}=2 \sin ^{2}\left(\frac{\pi \sigma}{2 \sigma_{0}}\right)
$$

The estimation of the crack-opening displacement $\phi$ was obtained by Goodier and Field. ${ }^{12}$ This can be written

$$
\phi=\frac{8 C \sigma_{0}}{\pi E} \ln \left\{\sec \left(\frac{\pi \sigma}{2 \sigma_{0}}\right)\right\}
$$


where $E$ is the Young's modulus of the material. For small $\sigma / \sigma_{0}$ these expressions are simplified to

$$
\begin{gathered}
s=\frac{\pi}{8}\left(\frac{K}{\sigma_{0}}\right)^{2} \\
\phi=\frac{K^{2}}{8 E \sigma}
\end{gathered}
$$

where $K$ is the stress intensity factor.

\section{EXPERIMENTAL}

The specimens were cut along the machine direction from extruded polycarbonate film $39 \mu$ thick and nylon 6 film $41 \mu$ thick as rectangular strips, $20 \mathrm{~mm}$ wide and $150 \mathrm{~mm}$ long. All specimens were used without annealing so that they were slightly anisotropic. Figure 2 shows their stress-strain characteristics. Yield stresses used for the calculation in the following experiments and obtained from the stress-maximum for polycarbonate and the tangential intersection for nylon, were 5.5 and $2.0 \mathrm{~kg} / \mathrm{mm}^{2}$, respectively.

In the center of one edge of the specimen, a

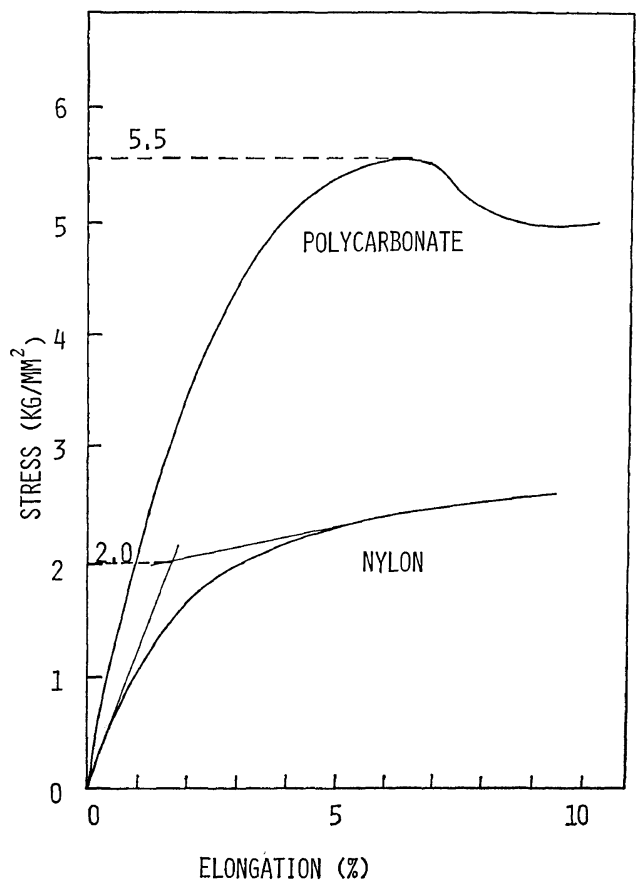

Figure 2. Stress-strain curves at $20^{\circ} \mathrm{C}(1.08 \% / \mathrm{min})$ for specimens of polycarbonate and nylon films. The values denote yield stresses.

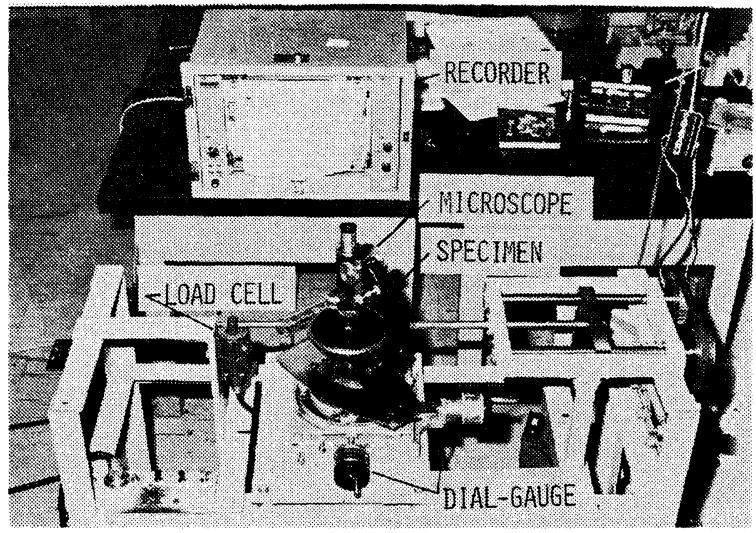

Figure 3. Testing apparatus.

sharp crack of controlled length was introduced by driving a new razer blade while it was held under a slight strain. The top radius of a crack induced in this way was found to be not larger than $1 \mu$ and there was no evidence of residual stress at the crack tip.

As shown in Figure 3, the tensile apparatus is suitable for using a polarizing microscope. The microscope can be moved in the two orthogonal directions and the distance of the movements can be read by use of dial-gauges within $0.1 \mu$. The specimen was mounted horizontally between two clamps. To one of the clamps was attached a tensile load-cell (ToyoBaldwin, UK-2K-120) and the output was recorded on a Model SS-505D recorder (ToyoBaldwin). The specimens were extended at a strain of this stress and the plastic-zone sizes were measured by use of both the dial-gauges and the microscope. All measurements were made in air at $20^{\circ} \mathrm{C}$ and $65 \% \mathrm{RH}$.

\section{RESULTS}

\section{Plastic-Zone Profiles}

The successive steps of the plastic deformation occurring ahead of a crack tip in nylon and polycarbonate are shown in Figure 4 . In both cases, as may be seen from these microphotographs, the shapes of the plastic-zone show wedge-type characteristics, and these shapes become clearly visible when the slow cracking at the crack tip initiates. Alternatively, there seems to be a threshold stress below which neither plastic deformation nor stable cracking 
would occur. The boundary of these plasticzones consists of a somewhat black and rather broad line. This is the elastic-plastic transition region where the materials are incompletely deformed. To find the plastic-zone dimensions, the inside of the contours was measured.

One of the well known and widely adopted criteria for predicting a yield boundary was proposed by von Mises ${ }^{13}$ in 1913. The Mises criterion is essentially a strain energy criterion for instability of the stress field, and it is expressed in principal stress forms as follows.

$$
\left(\sigma_{1}-\sigma_{2}\right)^{2}+\left(\sigma_{2}-\sigma_{3}\right)^{2}+\left(\sigma_{3}-\sigma_{1}\right)^{2}=2 \sigma_{0}{ }^{2}
$$

where $\sigma_{1}, \sigma_{2}$, and $\sigma_{3}$ are the principal stresses, and $\sigma_{0}$ is the yield stress under uniaxial tension. If attention is confined to the stress field in the immediate vicinity of the crack tip in a twodimensional body, the principal stresses associated with a polar coordinate system with an origin at the tip of the crack are given ${ }^{14}$ by

$$
\begin{aligned}
& \sigma_{1}=\frac{\sigma \sqrt{\pi C}}{\sqrt{2 r}} \cos \frac{\theta}{2}\left(1+\sin \frac{\theta}{2}\right) \\
& \sigma_{2}=\frac{\sigma \sqrt{\pi C}}{\sqrt{2 r}} \cos \frac{\theta}{2}\left(1-\sin \frac{\theta}{2}\right) \\
& \sigma_{3}=0
\end{aligned}
$$

where $\theta$ is the angle between the axis of the crack, $r$ is the radius vector, and $C$ is half of the crack length. Applying these principal stresses to the von Mises criterion, eq 5, we find the resultant elastic-plastic contour ahead of the crack tip to be

$$
r=\frac{\sigma^{2} \pi C}{2 \sigma_{0}{ }^{2}} \cos ^{2} \frac{\theta}{2}\left(1+3 \sin ^{2} \frac{\theta}{2}\right)
$$

Figure 5 shows this result in dimensionless form. As is evident by comparing Figure 5 with Figure 4 , the shapes of the plastic-zone actually obtained with polycarbonate and nylon show no resemblance to the elastic-plastic contour predicted by the von Mises criterion, although the development of yielding in nylon is more elongated to the stress direction than in polycarbonate.

If observation is confined to the isochromatic fringes around the crack tip, the appearance has a certain similarity to the contours derived from the von Mises criterion, as shown in Figure 6. Vincent ${ }^{15}$ has obtained a photograph

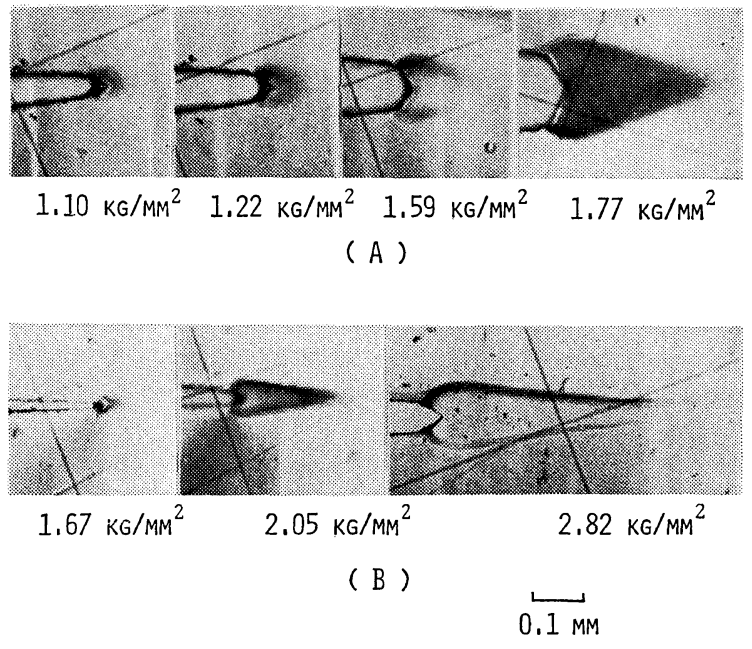

Figure 4. Successive plastic-zone growth near the crack tip in stressed specimens of (A) nylon and (B) polycarbonate.

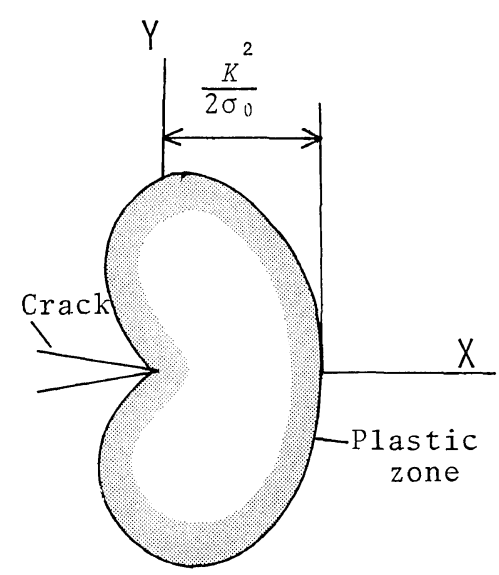

Figure 5. Elastic-plastic boundary near the crack tip obtained from the von Mises criterion.

similar to that in Figure 6, and he concluded that this type of plastic-shape was characteristic in the polycarbonate in the earliest stages of straining. However, careful consideration should be given to this suggestion, because the isochromatic fringes connot exactly reflect the plasticzone contours. By examining Figure 7 closely, which is the same part as in Figure 6 being photographed with a normal microscope, the reader will note an appearance of the plasticzone which is clearly different from the isochromatic fringes. Furthermore, craze initiates 
Fracture Processes in Ductile Polymers. I.

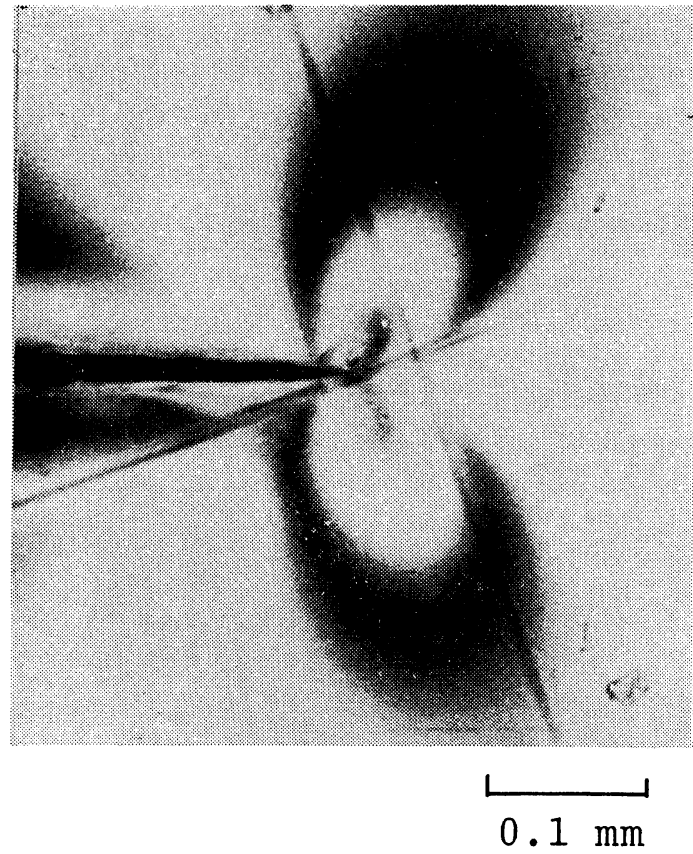

Figure 6. Isochromatic fringes near the crack tip in polycarbonate.

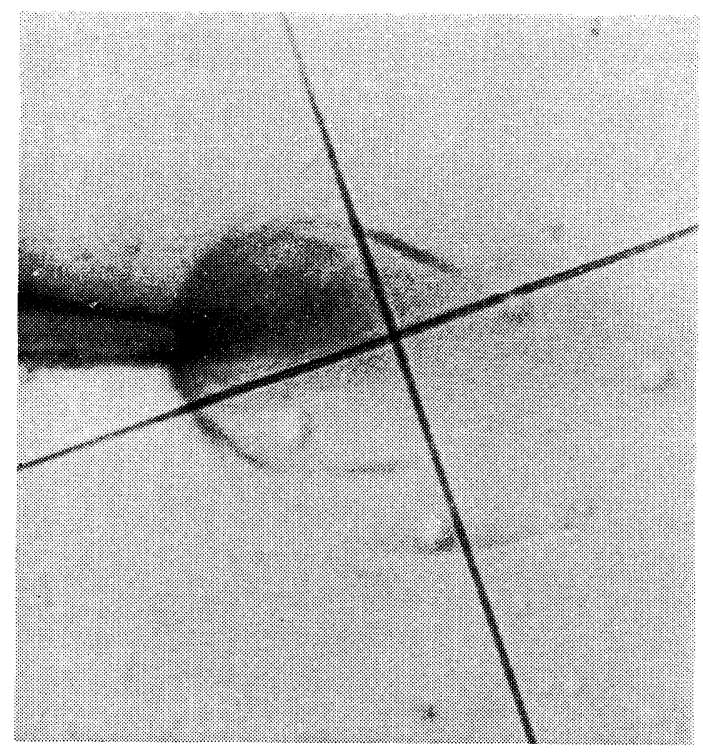

\section{$0.025 \mathrm{~mm}$}

Figure 7. Normal photograph of the same part as in Figure 6. at the crack tip in the initial stage of straining. As also may be observed from Figure 7, it is actually difficult to define the contour of the plastic-region in this stage, because the reduction in the thickness of this region is obscure. Therefore, the plastic-zone sizes were measured after a certain stage in which the shape started to resemble the Dugdale type yielding. This corresponds to the stress or strain above the critical value, as mentioned before. A more detailed discussion about this point, combined with the structure of the plastic-zone, will be given in the second paper of this series.

Plastic-Zone Sizes

The results of the measured plastic-zone length and crack-opening displacement in polycarbonate samples of different crack sizes are shown in Figures 8 and 9, respectively. The broken lines in these figures are those corresponding to the theoretical relations calculated from eq 1 and 2 . For the purpose of comparison, on the figures are represented the plastic lengths which were divided by the initial and current crack length. In these figures there is considerable scatter, part of which seems to be attributable to the deviation of the crack shape from the ideal form, but the variations in the experimental details do not produce any significant change in the general comparison of agreement with the theoretical curves.

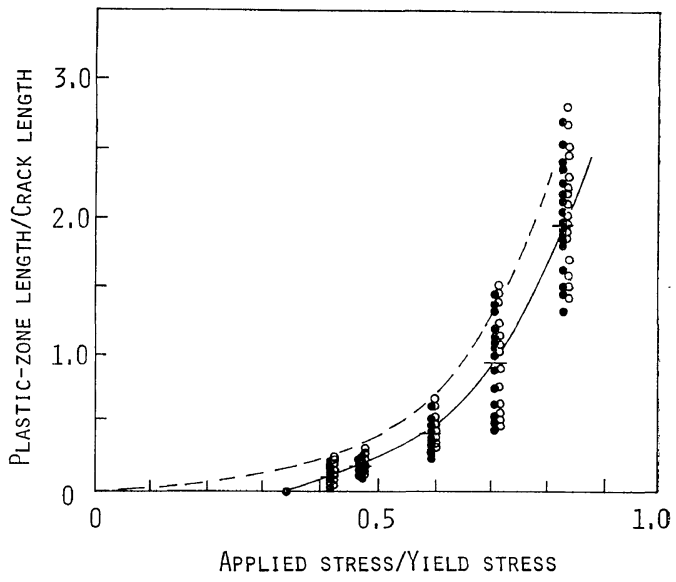

Figure 8. Camparison of the plastic-zone length in polycarbonate to the Dugdale theory (broken line): $O$, divided by current crack length; $\bigcirc$, divided by initial crack length. 


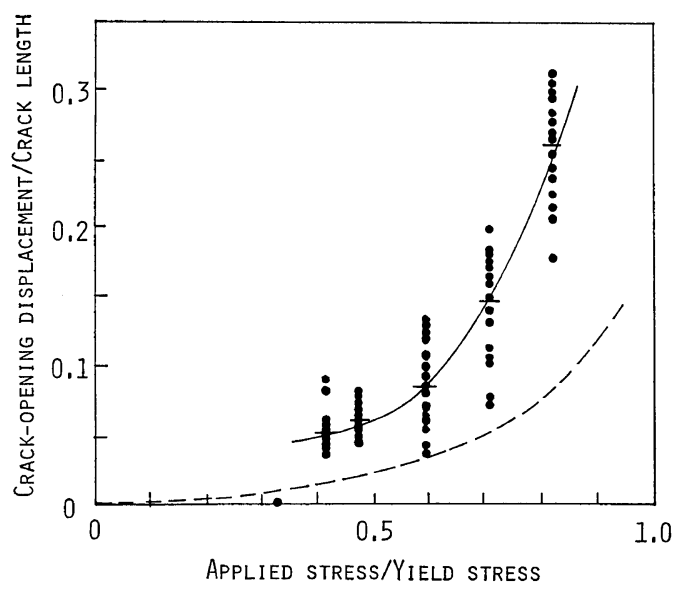

Figure 9. Comparison of crack-opening displacement in polycarbonate to the Dugdale theory (broken line).

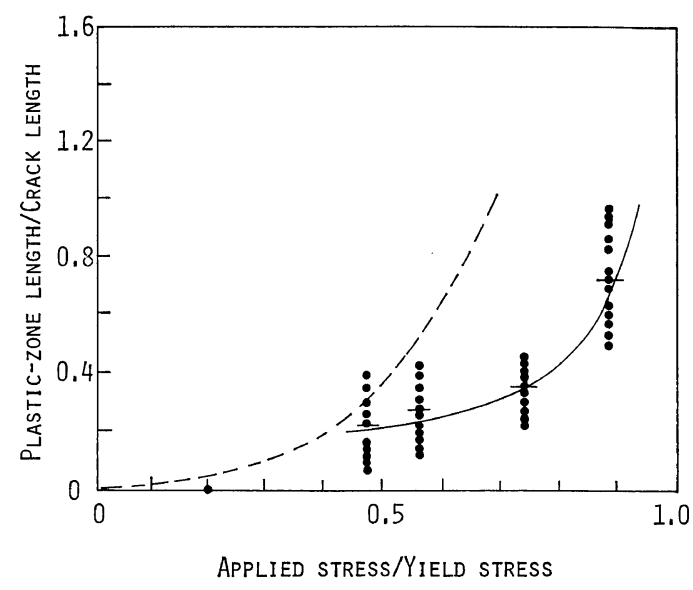

Figure 10. Comparison of plastic-zone length in nylon to the Dugdale theory (broken line).

In both cases it will be observed that the experimental data do not compare so well with the Dugdale theory. In general, the plastic-zone grows less and crack opens more than those predicted by the Dugdale theory. If attention is confined to the plastic-zone length in Figure 8 , the experimental data will be in agreement with the theory by shifting them parallel to the left. Alternatively, this clearly suggests that there is a threshold stress below which plastic yielding would not occur.

As shown in Figures 10 and 11, the results obtained with nylon give a more clear disagree-

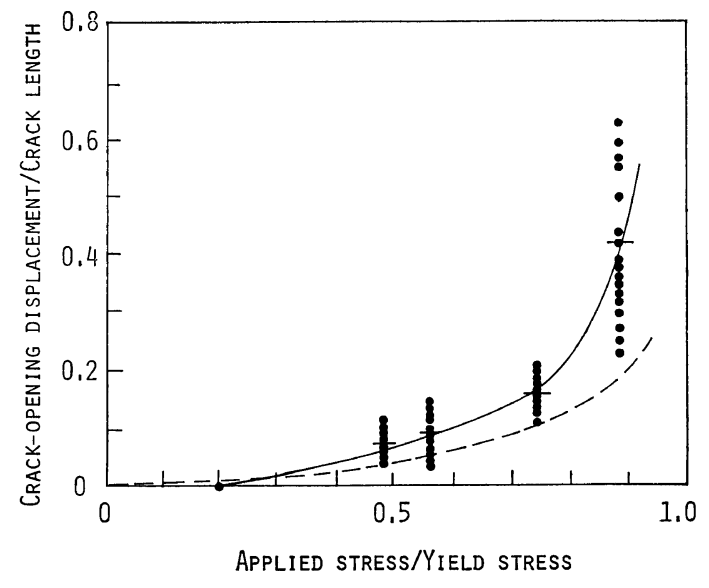

Figure 11. Comparison of crack-opening displacement in nylon to the Dugdale theory (broken line).

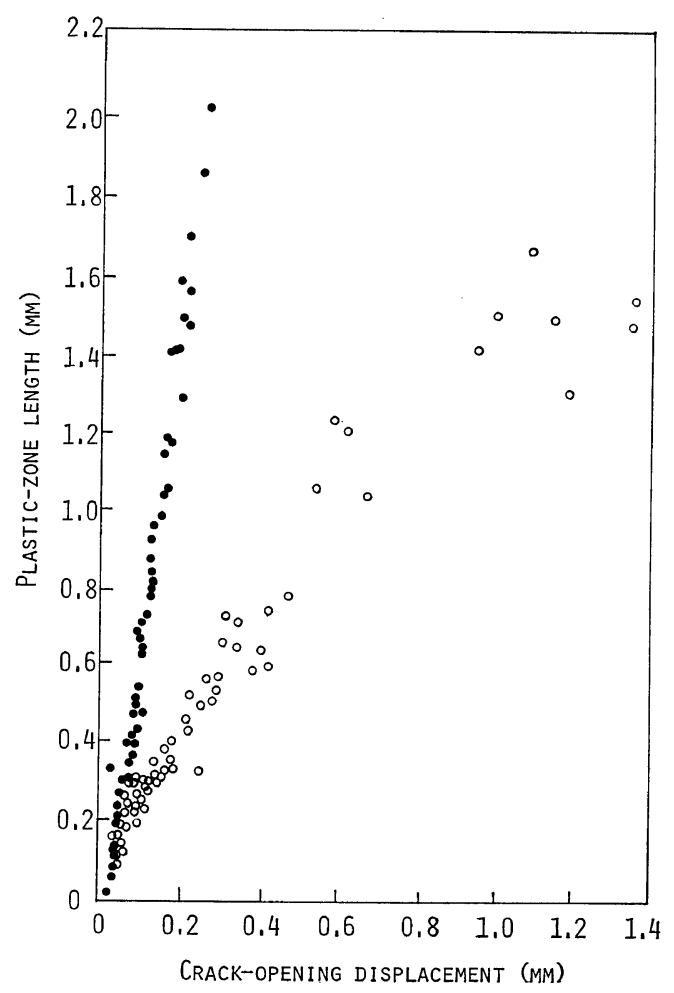

Figure 12. Relation between crack-opening displacement and plastic-zone length for (O) polycarbonate and $(\bigcirc)$ nylon.

ment between the experimental data and Dugdale predictions. The plastic-zone shape of the nylon shows the tendency to grow more easily in the 
Fracture Processes in Ductile Polymers. I.

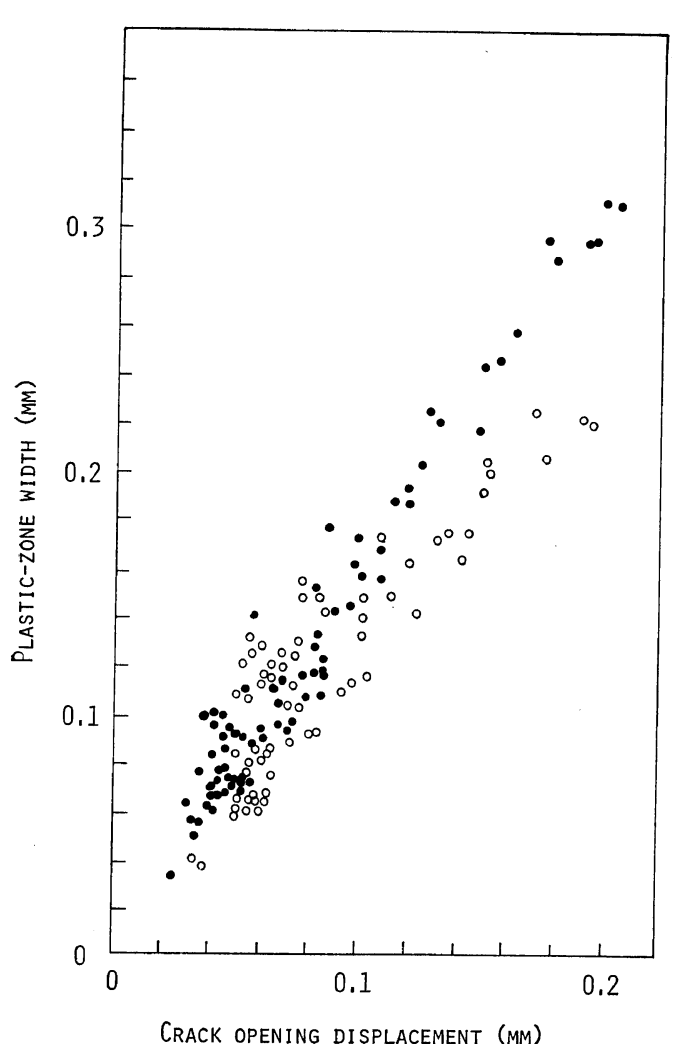

Figure 13. Relation between crack-opening displacement and plastic-zone width for (O) polycarbonate and $(\bigcirc)$ nylon.

direction normal to the crack than does polycarbonate. According to the Dugdale theory, the plastic-zone length should be proportional to the exponent of $\phi$ as derived from eq 2, but as shown in Figure 12, the data obtained with both the polycarbonate and nylon are in linear proportion to their crack-opening displacements. It is of interest that these relations apparently agree with those of small scale yielding, which is the first approximation of the Dugdale theory (see eq 3 and 4).

As shown in Figure 13, it should be noted that the crack-opening displacement linearly corresponds to the plastic-zone width which was measured from the maximum extension of the plastic-zone. Therefore, in order to obtain a more realistic criterion for ductile fracture of this class of materials on the basis of energy balance concepts, the plastic work should be considered in excess of that predicted by the
Dugdale evaluation. ${ }^{16}$

\section{DISCUSSION AND CONCLUSIONS}

The results obtained here are somewhat different from those of Brinson. ${ }^{10} \mathrm{He}$ had also chosen polycarbonate film as a representative ductile polymer and measured the plastic-zone size from the isochromatic fringe pattern. However, he concluded that both the plasticzone length and the crack-opening displacement agreed well with the Dugdale predictions. Unfortunately, since there was no information given on the mechanical properties of the material used by him, the reason for the discrepancy between our experimental results and his is not known. However, the method by which one identifies the plastic-zone length with the discontinuity in the slope of the isochromatic fringes is likely to overestimate the length, because the isochromatic fringes in the plasticzone are apparently produced by the thickness gradient which is associated with the incomplete transition of elastic to plastic deformation.

Consequently, though the plastic-zone shapes generally compare well with the Dugdale type yielding, the results obtained here suggest that the theory cannot be exactly descriptive of the length and crack-opening displacement. Even if the study is limited to the two-dimensional model (plane stress or plane strain condition), there are, in practice, various factors that cause the plastic-zone sizes to deviate from the Dugdale predictions. Anelasticity, anisotropy, strain hardening effect, time and temperature dependence, and inhomogenity, in the strict sense, should be considered. Figure 14, as a typical example, shows the change in the plastic-zone length of the polycarbonate which was held at constant strain for a period of $1 \mathrm{hr}$.

However, no theory which involves all the factors mentioned above is available at present. In these circumstances, it is potentially more fruitful to modify the Dugdale model and to examine it by applying experimental correlations. Such a modification, where the cohesive stresses change along the crack surfaces, has been proposed by Bergkvist ${ }^{11}$ in order to analyse the plastic deformation in poly(vinyl chloride). According to him the cohesive stresses which 


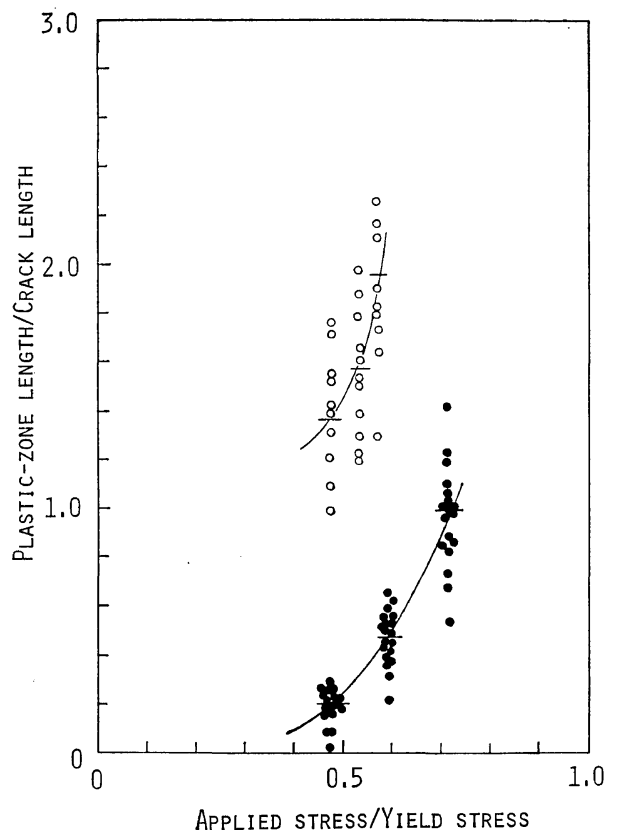

Figure 14. Time dependence of plastic-zone length for polycarbonate: immediately after stressing; $\mathrm{O}, 1 \mathrm{hr}$ after stressing.

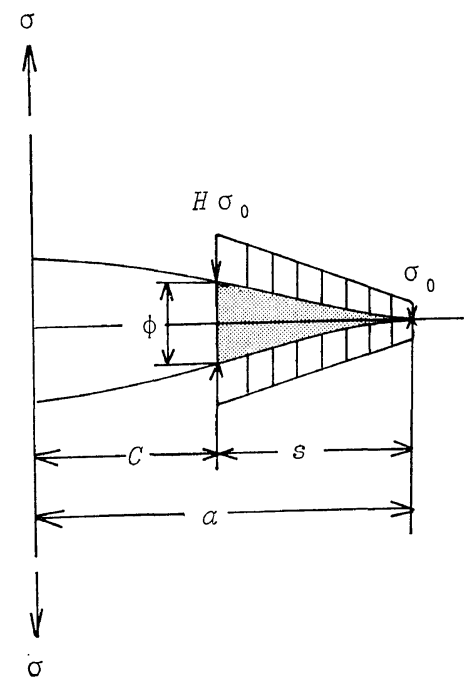

Figure 15. Modified Dugdale model: $H$, stress gradient parameter.

vary linearly are assumed as shown in Figure 15. This leads to the following expression for the plastic-zone length.

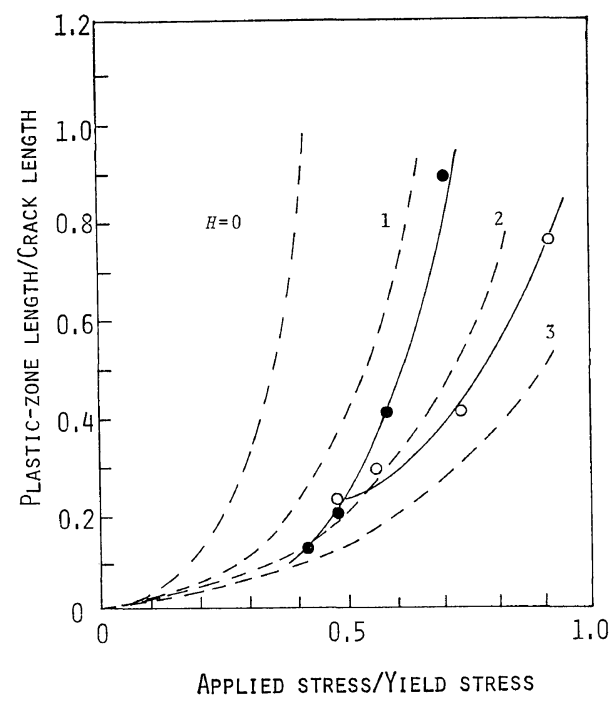

Figure 16. Comparison of plastic-zone length in (O) polycarbonate and $(\bigcirc)$ nylon to the modified Dugdale theory (broken lines).

$$
\begin{aligned}
\frac{\pi \sigma}{2 \sigma_{0}}= & \sin ^{-1}\left(\frac{1}{1+s / C}\right)-\frac{\pi}{2}+(1-H)\left\{\left(\frac{2+s / C}{s / C}\right)^{1 / 2}\right. \\
& \left.-\frac{1+s / C}{s / C}\left(\sin ^{1}\left(\frac{1}{1+s / C}\right)-\frac{\pi}{2}\right)\right\}
\end{aligned}
$$

Note that for $H=1.0$ the expression is simplified and give the usual expression of Dugdale.

Figure 16 shows the mean values which were obtained from Figures 8 and 10, and also the calculated curves which were obtained from eq 8 for typical values of $H$. The agreement between the experimental plots and the modified theory became rather reasonable and the values of $H$ are larger than 1.0 for both polymers. The values of $H$ which most accord with the experimental curves are 1.3 for polycarbonate and 2.5 for nylon. These results indicate that the cohesive stresses increase linearly with approach to the crack tip. This is quite different from the results of poly(vinyl chloride), which showed a value smaller than 1.0. Of course, such distributions of the cohesive stress should be verified from a different approach, such as detailed photo-elastic and plastic analyses. The assumption that the cohesive stresses, however, vary along the contour of the plastic-zone appears not unreasonable, because the thickness of the plastic-zone is strongly reduced with 
Fracture Processes in Ductile Polymers. I.

approach to the crack tip.

In closing, it should be mentioned that observations of the kind discussed here will be of use in analyzing a criterion for crack propagation in ductile polymers. They are also of use in understanding the details of energy absorption during plastic deformation prior to fracture initiation. Since the fracture and deformation problems in ductile polymers are highly complicated, in this way, with various factors, it is evident that more work using different approaches is necessary. One of the future studies would be to observe the structure and morphology of the plastic-zone; this will be reported subsequently.

Acknowledgment. The authors wish to thank Mr. Murayama for his assistance in the experimental work. The authors are also indebted to Teijin Co. for supplying polycarbonate and Unitika Co. for nylon 6 films.

\section{REFERENCES}

1. J. P. Berry, J. Polym. Sci., 50, 107 (1961).

2. G. R. Irwin, J. Appl. Mech., 29, 361 (1957).

3. G. P. Marshall, L. E. Culver, and J. G. Williams,
Plastics and Polymers, Feb., 75 (1969).

4. J. P. Elinck, J. C. Bauwens, and G. Homes, Intern. J. Fract. Mech., 7, 277 (1971).

5. G. P. Marshall, L. E. Culver, and J. G. Williams, Plastics and Polymers, April, 95 (1970).

6. I. Narisawa and T. Kondo, J. Polym. Sci. Phys. ed., 11, 223 (1973).

7. P. I. Vincent, Polymer, 12, 534 (1971).

8. R. J. Fergunson and J. G. Williams, ibid., 14, 103 (1973).

9. D. S. Dugdale, J. Mech. Phys. Solids, 8, 100 (1960).

10. H. F. Brinson, Exp. Mech., Feb., 72 (1970).

11. H. Bregkvist and H. Anderson, Intern. J. Fract. Mech., 8, 139 (1972).

12. T. N. Goodier and F. A. Field, "Fracture of Solids," Interscience, New York, N.Y., 1963, p 103.

13. R. von Mises, Nachr. Ges. Wiss. Gott., 582 (1913).

14. G. R. Irwin, "Handbuch der Physik, " SpringerVerlag, New York, N.Y., 1958, p 79.

15. P.I. Vincent, "Deformation and Fracture of High Polymers," Plenum Press, New York, N.Y., 1973, p 287.

16. T. Yokobori and A. Kamei, Intern. J. Fract. Mech., 7, 367 (1971). 